\title{
Immunopathology of Immune thrombocytopenia: Essentials for oncologists and haematologists
}

\author{
Piyush Vyas*
}

*Department of Haematology, Oncology and Internal Medicine, Medical University of Warsaw Hospital, Warsaw, Poland

Received: 8 October, 2017; Accepted: 26 October, 2017; Published: 16 November, 2017

*Corresponding author: Piyush Vyas, Department of Haematology, Oncology and Internal Medicine, Medical University of Warsaw Hospital, Warsaw, Poland; email: vyas_piyush20@yahoo.co.in

\begin{abstract}
An in depth knowledge is essential about the pathology of immune thrombocytopenias before we start managing these autoimmune disorders. It's important to differentiate whether the patient has primary or secondary immune thrombocytopenia. If the aetiology is known, we try to eliminate the antigen which triggers further formation of autoantibodies directed against platelets. If aetiology is unknown then as per international working group criteria, we diagnose such immune thrombocytopenia as primary immune thrombocytopenias. Primary immune thrombocytopenias are managed primarily by administering intravenous immunoglobulins or steroids as first line treatment. In second line immunosuppressive drugs like azathioprine, cyclosporine, rituximab, vinca alkaloids like vincristine, cyclophosphamide or other drugs like androgens- danazol, TPO mimetics etc. are administered. And of course for refractory or relapsed ITP splenectomy remains as an important surgical option however now-a-days less popular.

Keywords: ITP- immune thrombocytopenia; ALPS - Autoimmune lymphoproliferative Syndrome; SLE -Systemic lupus erythematosis; VZV- Varicella zoster virus; APS - Antiphospholipid syndrome; CVID - Common variable immune deficiency; MMR- Measles, mumps, rubella.; MDS -Myelodysplastic syndrome; GP- Glycoprotein; i.v.Ig - Intravenous immunoglobulins; TPO- Thrombopoeitin mimetics; NK-natural killer; MHC- Major histocompability Complex; APCantigen presenting cells; TCR - T cell recerptor; Tregs -T relulatory Lymphocytes ;IL-interleukin ; TGF-transforming growth factor; IDDMinsulin dependent diabetes mellitus ;CTL- cytotoxic T lymphocyte.
\end{abstract}

\section{Introduction}

Immune thrombocytopenia (ITP) are autoimmune disorders which characterize that autoantibody mediated platelet destruction, suboptimal platelet production and $\mathrm{T}$ cell mediated platelet lysis occurs leading to decrease in platelet count.

\section{Clinical Definition of primary and secondary immune thrombocytopenias}

As per the International working group criteria, primary ITP is clinically defined by the presence of thrombocytopenia in the absence of demonstrable causes be it be pathogenic, therapeutic, congenital, or systemic (autoimmune diseases like for example SLE) and a platelet count of less $100 \mathrm{G} / \mathrm{l}$. Thus a diagnosis of primary ITP can only be diagnosed by thoroughly examining a patient which would include detailed history taking, physical examination, full blood count and a bone marrow biopsy. The secondary ITP broadly include all the other forms of immune thrombocytopenia except the primary ITP.Secondary ITP can be $\mathrm{H}$. Pylori related, drug related, HIV related, related with autoimmune disorders like SLE etc..(1)

Of prime importance here is to differentiate primary immune thrombocytopenia with secondary immune thrombocytopenia are managed differently thus it should be assured that the patient be screened for HIV or HCV. A bone marrow aspiration and a trephine biopsy are further advised for all patients ( especially elderly patients $>60$ years) to rule of myelodysplastic syndromes (MDS) and haematological malignancies .Of course autoimmune diseases like SLE also can present as isolated thrombocytopenias as their initial stages thus such diseases should also be excluded. If patient has autoimmune haemolytic anaemia (AIHA), always Evans syndrome along with thrombocytopenic Evans syndrome can be suspected (2).

\section{Definition of different phases of ITP}

The International working group recommended the use of "newly diagnosed ITP" for all cases at diagnosis. The term "persistent ITP," was introduced for patients with ITP to define the period lasting between 3 and 12 months from diagnosis. This category includes patients not achieving spontaneous remission or not maintaining their response after stopping treatment between 3 and 12 months from diagnosis. The term "chronic ITP" was suggested for patients with ITP lasting for more than 12 months. Term "severe" ITP was reserved for ITP with "clinically relevant bleeding" which was defined by the presence of bleeding symptoms at presentation sufficient to mandate treatment, or by the occurrence of new bleeding symptoms requiring additional therapeutic intervention with a different platelet-enhancing agent or an increased dose. 


\section{Immune thrombocytopenias and solid tumours}

There have been isolated case studies or isolates case series reported of incidences of co-existent cancers especially solid tumours with immune thrombocytopenias. Our group was the first to publish a case report of a patient with metastatic duodenal cancer with coexistent immune thrombocytopenia (P.Vyas et.al.) (7). In this case we first treated the immune thrombocytopenia and when the platelet count of more than 75 G/l was achieved, we treated this patient with chemotherapy without any episodes of bleeding. Our observation showed as the cancer responded to treatment, the platelet count increased. Afterwards again we reported a case of breast cancer with coexistent immune thrombocytopenia\{P.Vyas et.al. (9)\} where initially the patient was treated with endocrine therapies, and when the disease got endocrine refractory, chemotherapy was administered successfully achieving long periods of stable disease. As far as ITP is concerned our patient was initially treated with steroids and immunoglobulins, when the immune thrombocytopenia got refractory to immunoglobulins and steroids, treatment with rituximab, vincristine, danazol was administered with good effects, attaining satisfactory levels of platelet counts to further treat the patient with chemotherapy. Since these 2 disease entities(immune thrombocytopenia and solid tumours) occur rarely or rarely are diagnosed as immune thrombocytopenias with coexistent solid tumours, it is difficult to conduct prospective randomised clinical trials.Thus we treat these patients on the basis of available knowledge in literature .It seems that many patients with immune thrombocytopenias with coexistent solid tumours are not diagnosed by oncologists , as chronic thrombocytopenia in these cases is misunderstood as massive tumour infiltration to the bone marrow. Many a times trephine biopsy is not done to find out the reason of thrombocytopenia / cytopenias. Isolated thrombocytopenia if it occurs in a patient should not be understood a priori as infiltration by tumour unless we find massive tumour infiltration in bone marrow biopsy. Many of these patients with solid tumours with coexistent chronic thrombocytopenia have chronic immune thrombocytopenia which get resolved with effective treatment of immune thrombocytopenias with steroids or intravenous immunoglobulins and with effective anti cancer therapies, be it surgery or chemotherapy or other treatment modalities .Case studies have shown remission of immune thrombocytopenias by effective treatments. Not every patient with thrombocytopenia should be disqualified from potential curative or life prolonging palliative therapies. In our e-poster at Polish Association of Haematologists and transfusion medicine specialists Conference, Warsaw , 2017\{P.Vyas et.at.(16)\} we presented case reports of our patients who had initially immune thrombocytopenia, and after effective treatments not only the patients had effective anti-neoplastic therapy, but their platelet count too increased to satisfactory levels. Our observation showed that with effective anti-neoplastic therapies especially with use of immunosuppressive drugs like cyclophosphamide or vincristine, it is possible to effectively treat and cure/prolong life of cancer patients with coexistent thrombocytopenias. Most of the cases of immune thrombocytopenias coexisted with breast, lung cancers, renal cancers, with prostate cancer even rarely.
Opinions are very conflicting, some authors advocate that these are paraneoplastic phenomena of cancers \{Krauth et.al. $2012(17)\}$, others are of the opinion that no correlation exists between these 2 diseases if they coexist. Whether there exists some significant correlation between immune thrombocytopenias and cancers remains still is a topic of further research.

Some of the proposals about treating these solid tumour patients with coexistent immune thrombocytopenias are that of using protocols with combined chemotherapy drugs (21) like cyclophosphamide, vincristine, vinblastine, cyclosporine, procarbazine, etoposide, steroids in their regimens. In cases where radical resection is possible (ovarian cancers, breast cancers, lung cancers etc.) it is advised to resect radically the tumour after attaining an optimal platelet count from ITP directed therapies (steroids/immunoglobulins etc.). In referactory cases splenectomy is an option now-a-days less popular among physicians and patients too.Since endocrine therapies can be administered safely in thrombocytopenias, we recommend to start treatment with endocrine therapies for example in breast cancer (ER/PR + patients), prostate cancer patients. Our observations found that protocols like FOLFOX/ FOLFIRI / Capecitabine can be safely administered in gastrointestinal cancer patients. Protocols like AC (Doxorubicine, Cyclophosphamide) /Docetaxel monotherapy /Cyclophosphamide monotherapy / Vincristine monotherapy can be safely administered in breast cancer patients with coexistent immune thrombocytopenias. In a situation when no guidelines exists, we recommend to treat patients as have been done the world over based on case studies/ case series as far as solid tumors with coexistent ITP is concerned.

\section{Immune thrombocytopenias and Haematological malignancies}

Certain protocols are advised if immune thrombocytopenias occur with haematological malignancies. In chronic lymphocytic leukaemia where the incidence of coexisting autoimmune diseases is high, among others autoimmune haemolytic anaemia, autoimmune immune thrombocytopenia and of Evan's syndrome some authors have suggested administering protocols like Rituximab-Cyclophosphamide-Dexamethasone combination in the management of autoimmune cytopenias associated with chronic lymphocytic leukemia (18). It has been noted that there exists an increases incidence of non Hodgkin lymphomas in certain autoimmune disorders as shown in pooled analysis within the InterLymph Consortium (19). In various case studies splenectomy or steroids was advised for the treatment of Hodgkin lymphoma patients (20). In haematological malignancies especially CD20+ B cell lymphomas multi drug protocols with rituximab is a valuable option not only for treating lymphomas but also immune thrombocytopenias.

\section{Differential diagnosis}

Differential diagnosis of thrombocytopenias includes many diseases states where thrombocytopenia occurs. Many a times we have so called pseudothrombocytopenia which occurs in $1: 1000$ individuals, in about $15 \%$ of ambulatory patients with 
thrombocytopenia. In pseudothrombocytopenia -platelet clumping occurs where EDTA dependent IgG plays a role .Occurs when platelets with neoepitope are exposed in presence of EDTA. Occurs in EDTA containing tubes. In such cases use of citrates or heparins will give the actual count. Another condition like dilutional thrombocytopenia occurs in patients who get massive amounts of crystalloids or colloids.

Thrombocytopenia can be congenital or acquired. In congenital thrombocytopenias, patients have a family history of thrombocytopenia, may present in adulthood, often are associated with skeletal abnormalities, change in platelet size and change in platelet function. Isolated acquired hypoproliferative thrombocytopenia can be caused by infections like HIV, HBV, CMV; with drugs like linezolid, bortezomib; by alcohol and also in MDS(5-10\% of thrombocytopenia in MDS is isolated thrombocytopenia).Isolated thrombocytopenias can occur due to processes which involve increased destruction of platelets like immune or non immune thrombocytopenias .Immune thrombocytopenias can be alloimmune thrombocytopenia which occurs in for example post transfusion purpura or in isoimmune neonatal thrombocytopenia. Autoimmune thrombocytopenia can be primary ITP or secondary ITP.

Other causes of thrombocytopenias are for example heparin induced thrombocytopenias or other drug induced immune thrombocytopenias, now classified as secondary immune thrombocytopenias ( for example after therapy with quinine, gold etc.).

Non immune thrombocytopenias due to increased destruction of platelets occur in diseases like disseminated intravascular coagulation,sepsis,may occur in the use of extracorporal circulation, can occur in post operative periods after surgery and also in patients with large clot burden for example in pulmonary embolism, in thrombotic microangiopathies (TTP,HUS).

Thrombocytopenia may occur in disease states with increased sequestration.In normal conditions only $1 / 3$ of platelets are stored in spleen and $2 / 3$ of platelets is circulating in peripheral blood. In states where splenomegaly occurs increased sequestration of platelets occurs. Splenomegaly occurs in various disease states for example in chronic liver disease.

As far as immune thrombocytopeni are concerned $80 \%$ of cases are primary ITP and $20 \%$ of cases are secondary ITP. Secondary ITP occurs in various disease states like SLE, APS, in limphoproliferative disorders like CLL, H.Pylori and also seen after vaccinations for example after MMR (Measles mumps rubella) vaccination.

\section{Platelet Physiology}

To understand pathogenesis of ITP, a clinician should have good understanding of platelet physiology. Platelets play an important role in haemostasis, they prevent and arrest hemorrhage. Platelet membrane Glycoproteins (GP) act as physiological receptors and play a role in interacting between vascular subendothelium and the platelets (adhesion) and platelet platelet interaction (aggregation). A single megakaryocyte can produce several thousand platelets. Daily $2 \times 10^{11}$ platelets are produced. Thrombopoetin regulates megakaryopoeisis and thrombopoiesis. Under normal conditions two third of total platelet mass is circulating at concentration of 150 to $400 \mathrm{G} / \mathrm{l}$, the rest remains in splenic circulation.

Under physiological conditions platelets survive, have a lifespan of 7 to 10 days, after which are sequestrated in spleen. However liver and accessory spleens also play role in platelet destruction. Under conditions where destruction of platelets occur for example by autoantibody the mean life span of platelets is significantly reduced.

\section{Platelet membrane glycoproteins}

GP IIb IIIa and GP Ib IX complexes are the most immunogenic of the platelet glycoproteins, carrying some recognized autoepitomes. GP IIb IIIa complex is a member of the superfamily of integrins.GP IIb IIIa complex is required for normal platelet aggregation .GP IIB IIIa complex appears very early at promegakaryocyte stage and is considered as early marker of megakaryocytic lineage. When activated GP IIaIIIA clustering occurs .GP Ib IX V complex plays an important role in the adhesion of platelets to exposed vascular subendothelium. Along with Von Willebrand factor, this complex causes platelet activation which promotes the recruitment of additional platelets .GP Ia IIa complex is also a member of integrin superfamily of adhesion receptors (alpha2beta1). GP Ia IIa complex functions as a receptor for collagen, and mediates as a significant portion of the adherence of platelets to the subendothelium.

\section{Fc gamma receptors}

Fc Fragment of IgG receptors are probably the binding sites for phagocytes, monocytes, macrophages, neutrophils, eosinophils and also platelets .Activation of FcR receptors induces activation of phagocytes which further causes release of inflammatory mediators. Fc Gamma R type I, FcGamma R type II, Fc Gamma R III are the 3 different subclasses of this receptor. The only Fc Gamma $\mathrm{R}$ expressed on platelet is Fc Gamma R IIA.

\section{Autoimmune Thrombocytopenia}

If our body's own antigen is recognized as foreign entity, autoimmune disease occurs. Autoimmune disease may be organ specific or can manifest as a systemic disorder. Lymphocytes $\mathrm{T}$ and lymphocytes B play an important role in autoimmune disorders. The mechanism is very complex, however an oversimplified version would be that lymphocytes $\mathrm{T}$ especially $\mathrm{T}$ regulator cells influence the production of autoantibodies being produced by lymphocyte $\mathrm{B}$ cells. In autoimmune thrombocytopenia autoantibodies are produced against platelets resulting in platelet sequestration mainly in spleen. It seems that in some populations ITP is much common, in other populations the disease is rare, as HLA molecules too play a role in autoimmunity. Different HLA molecules have different peptide binding properties and are selective for particular T cells. Thus in certain populations with a particular HLA setup, certain autoimmune diseases are much more manifested. 


\section{Immunology of autoimmune disorders}

Our body has 2 immune systems, the innate immunity and the adaptive immunity. Innate immunity is very ancient, found in a lot of vertebrates. It consists of epithelial barriers, the phagocytes ie. the neutrophils, macrophages, etc..Other components like complement system and NK cells take part in first line of defence ie. through innate immunity. Innate immunity actively defends our body mostly for 1 st 12 hours after infection, then comes the role of the so called "adaptive immunity". In adaptive immunity the B lymphocytes and T lymphocytes take part and defend our body against various infections. The B lymphocytes then transform to plasmocytes and produce antibodies, and the T lymphocytes ofcourse orchestrate the whole adaptive immunity for example via effector T cells. In humans, it seems this "adaptive immunity" plays much more important role in fighting pathogens. Adaptive immunity is the most important immune system in the hierarchy of immune systems in our body.

$\mathrm{T}$ cells bind to MHC class I molecules expressed on most nucleated cells and platelets (CD8+T cells) and the CD4+T cells bind to MHC II molecules expressed on APCs, macrophages, B cells and on activated Tcells. T cells become activated by antigen presenting cells like macrophages, dendritic cells etc.. In this process the first incident is the binding of the peptide on for example mature dendritic cell (APC) -MHC II molecule with T cell receptor (TCR) and binding of $\mathrm{B} 7$ peptide of mature dendritic cells to CD28 proteins of activated T helper cells. The process is much complex involving co-stimulatory signals, antigen specific TCR signals, CD28, CD80, CD86, CD40 ligand , CD40 proteins, CD3, alpha, beta TCR peptide antigen and MHC class II molecules. CD40 ligand and CD28 have to interact with their appropriate receptors on the antigen presenting cells surface. In autoimmune diseases the $\mathrm{T}$ cells have to be activated by this synapse. For autoreactive $\mathrm{T}$ cells to be activated, all of these synapses should be formed within our body.

Cytokine driven T cell activation also plays an important role in our body's immune system. After the two signals are met at the interface of $\mathrm{T}$ cell and antigen presenting cells, then the next most important processes which occurs afterwards is through cytokine network. The Th1 generates interferon gamma, Th2 generates IL4 resulting in good anti-inflammatory responses. Thus it is the interaction between Th1 and Th2 which would tell us amount of particular response to a particular antigen. The processes involve IL-4, interferon gamma, IL-12, IL-18, IL10, TGF-beta. Regulatory T cells have gained attention in last 2 decades, play a very important role in the whole process too. They are part od CD4 positive repertoire. Cytokines produced by Th1 cells and Th2 cells can downregulate each other for example IFN gamma and IL12 downregulate Th2 cells while IL10 inhibits Th1 cells CD4+CD25+Foxp3 T regulator cells mediate suppression by cell cell contact and by releasing cytokines (IL10 and TGF). Thus in regulatory $\mathrm{T}$ cell deficiencies, autoimmune diseases or allergic responses occur in our body. These $\mathrm{T}$ regs are produced in thymus (natural Tregs) or in peripheral blood in response to (induced Tregs) during immune responses.
By and large, $\mathrm{T}$ regs cause immunosuppression by(a) releasing inhibitory cytokines for example TGF beta(most immunosuppressive), IL35, IL10 which then act on effector T cells (b) by causing cytolysis - where granzyme A or granzyme $\mathrm{B}$ attack porforin pore thus causing apoptosis of effector T cells (c)by metabolic disruption caused by contact inhibition where CD39;CD73;IL-2 ;CD25 proteins take part causing death due to cytokine deprivation (d)and lastly by targeting dendritic cells via inhibition od DC maturation and function. In this process CTLA-4; CD80/CD86; LAG3; MHC class II proteins take part. And as a result the dendritic cells cannot present antigen and are inactivated.

Thus "autoimmunity" is immune reactivity against our own body. Autoimmunity can be organ specific or systemic (non organ specific). Examples of organ specific autoimmune diseases are multiple sclerosis(brain), pernicious anaemia(stomach),thyroid diseases like Hashimoto thyroiditis, primary myxoedema, thyrotoxicosis; adrenal gland diseases like Addison's disease; diseases like IDDM(pancreas autoimmune disorder)or autoimmune disorders like ITP. Examples of non organ specific autoimmune disorders are RA (joints),SLE, scleroderma, dermatomyositis.

For development of autoimmune disease certain requirements are to be met. Immune dysregulation; abnormal activation of dendritic cells, abnormal activity of $T$ cells and $B$ cells are some pathological immune processes going on in the autoimmune disorders. Also genetic and environmental factors like exposures to infectious agents too play an important role.

Since our topic is pathogenesis of ITP, we would talk in more detail about organ specific autoimmunity. Organ specific autoimmunity is deficiency of central or /and peripheral tolerance induction mechanism ie. the ability to eliminate or deactivate self reactive lymphocytes. The CD4+CD25+FoxP3 regulatory $\mathrm{T}$ cells suppress $\mathrm{T}$ cell functions. And in many autoimmune diseases the $\mathrm{T}$ cells cannot be suppressed and thus the disorder is manifested. In ITP, the autoantibodies are primarily IgG directed against epitopes on GP IIbIIIa (CD41) and/or GP IbIX(CD42). The complex immunopathology involves opsonisation of platelets, after which the platelets interacts with Fc gamma receptor of the macrophage which allow phagocytosis of the platelets. After phagocytosis of platelets, some of the proteins of the platelets are reprocessed and expressed on MHC type II proteins, which turns on autoreactive B cells, which then turn on other autoreactive B cells to generate more and more antibodies. At last this vicious cycle continues causing further platelet destruction.In some cases of ITP, we cannot demonstrate the formation of autoantibodies, the mechanism proposed here is that such patients have CD8+cytotoxic T cells and cause destruction of platelets by binding to megakaryocytes via CTL mediated mechanism. Thus a lot of heterogeneity of autoimmune pathogenesis are probably the cause of platelet destruction in ITP and not a single mechanism.

B cells play a central role in inducing autoimmunity. B cells differentiate to mature plasma cells which produce 
autoantibodies. Antibodies have heavy chains and light chains. About 6000 types of heavy chains can be produced. The light chains -kappa and lambda chains also have various types. There exist about 200 kappa chains and 120 possible lambda chains, put together it comes out to be about 320 different types of light chains. Thus when we combine the probable combinations of light and heavy chains ie 6000 heavy chains and 320 light chains, that comes out to be 1.9 million antibodies. Moreover, more diversity with splicing variations due to addition and deletions of nucleotide bases of DNA is possible with additional 30 million types of various antibodies produced. Now if we have 1.9 million antibodies combination of heavy and light chains; and 30 million variations of antibodies due to addition or deletion of nucleotide bases, then we can probably have 60 trillion probable antibodies types which can be produced in our body. Further, somatic mutations can introduce $3 \times 10^{13}$ various types of antibodies which together makes about $2 \times 10^{27}$ various possible antibodies in a human body.

Thus our body under normal regulatory mechanisms like clonal deletion (apoptosis), receptor editing, anergy (reduced membrane $\operatorname{IgM}$ ) and clonal ignorance (normal phenotype, no antibody secretion) makes these B cells dormant / inactive to avoid self reactivity.

Autoantigens stimulate "ignorant" cells. The B cells with specificity for DNA bind soluble fragments of DNA, sending a signal through B cell receptor. Then the cross linked B cell receptor is internalized with the bound DNA molecule. After which the GP rich fragments from the DNA which is internalized bind to TLR-9 in an endosomal compartment, sending a co-stimulatory signal. And thus these "ignorant" B cells are then activated and produce "autoantibodies". Different mechanisms are suggested for the formation of autoantibodies. One mechanism is formation of autoantibodies directly which occurs probably in ITP.B cells are APC, so these cells can directly ingest a platelet, process it, present platelet antigen peptides on it's surface which further causes clonal production of T cells. And these T cells further orchestrate next B cells to produce autoantibodies. This mechanism is called epitope spreading. Also B cells secrete cytokines; they can stimulate dendritic cells which enhance their antigen penetration again to next B cells. And thus in autoimmune disorders this vicious cycle starts when normal immune regulatory mechanisms fail.

In ITP, autoantibodies specific to GP IIb/IIIa (Fibrinogen receptor), GP Ib/IX (Von Willebrand receptor), GP Ia/IIa (collagen receptor) etc. are produced. Since serological assays have low specificity and sensitivity, these tests do not have a definitive role in making the diagnosis which till date as per the definition of International working group definition remains one of exclusion as far as primary immune thrombocytopenia is concerned. Thus in ITP, loss of self tolerance occurs. This can be due to immunodeficiency states like CVID, APLS; due to molecular mimicry (cross reactive antibodies to HIV, HCV,VZV) or /and due to disturbances in our immune repertoire like increased autoantigen presentation, epitope spreading, decreased $\mathrm{T}$ regs, decreased TGF beta cytokines, production of proinflammatory cytokines by exogenous antigens.

\section{Pathogenesis of Immune Trombocytopenias}

In the year 1951, Harrington et. al. (3) injected blood from patients with primary ITP into compatible healthy volunteers leading in marked reduction in platelet counts within hours. Thus these authors hypothesized existence of an anti platelet factor in the globulin fraction of plasma of patients diagnosed with primary ITP. Afterwards Mc Milan et al. Dixon et al. successfully described this factor as antiplatelet antibodies. Basically these are autoantibodies to platelet glycoproteins (GP)IIb/IIIa and GP Ib/IX; but autoantibodies to platelet glycoprotein GP Ia/IIa; GPIV, GPV have also been observed. The chief class of these antibodies is immunoglobulin (Ig)G, the complement fixing IgG1 and IGg3 isotypes. Researchers still do not know the underlying defect leading to these autoantibody production, but it is likely that more than one aetiology is involved. Infections causing molecular mimicry and epitome spread; also to a lower extend hereditability (as shown in PARC - ITP study; about 2, 3\% of children with ITP have family history of ITP) seems to be the autoantibody production triggering factors.

ITP with infectious aetiology have been reported by many authors .For example Murphy et.al. (1987) reported incidence of ITP among 10, 5\% of HIV patients. Similarly Kaslow et. al. (1987) reported 6, 7\%; Rossi et.al.(1990) reported 10, 9\%; Sullivan et.al. (1987) reported 8, 7\% and Mientjes et.al. (1992) reported an incidence of $23,5 \%$ of ITP among HIV positive patients. Thus a clear increase of incidence of ITP among HIV patients has been observed. Most of these patients have platelet counts less than 50G/l. In HIV associated ITP, a particular autoantibody is produced in these patients to a very specific aminoacids aa49-46 of GP IIIa molecule. And when these very specific autoantibodies touch GP IIIa molecules, they turn on 12 lipoxygenase which in turn generate reactive oxygen radicals via NADPH oxidase particularly superoxides .And at last, as a result platelet "suicide" occurs via ROS (reactive oxygen species)mediated platelet destruction. Thus these HIV infected patients have peroxide mediated platelet lysis.

Immune thrombocytopenia post infection is also reported in HCV positive patients without overt liver disease ranged from 10\% (Pawlotsky) upto 36\% (Pivetti,1996).Garcia Suarez (2002) reported an incidence of $23 \%$ of ITP among HIV positive patients .These ITP patients with HCV positive patients. These ITP patients with HCV infection manifest generally bleeding at higher platelet counts, may respond to interferon gamma. Corticosteroids may increase viral reproduction among these patients.

H. Pylori and ITP have an association, a fact well established from years. Many centres have published multiple single centre studies where an effect of eradication therapy on platelet counts in Helicobacter Pylori infected patients with primary ITP has been noted. Some studies showed an increase of platelet count upto $100 \mathrm{G} / \mathrm{l}$ and more in about $40 \%$ patients ie a complete response was achieved using triple therapy for Helicobacter Pylori eradication. In about $50 \%$ of patients with H.Pylori infection and ITP, a satisfactory platelet count of $30 \mathrm{G} / \mathrm{l}$ or more was achieved and at least double the baseline platelet level was achieved after triple therapy of H.Pylori. Some patients have good 
response to triple therapy, others don't. What is the explanation to this heterogeneity in ITP response to eradication of H.Pylori? Well some authors have suggested the mutability of ITP. Clonal evolution, epitome spread modify natural history of ITP. 1st generation antibody production in early stages, in later stages 2nd and even 3rd generation autoantibody production occurs which leads to non responsive to triple therapy disease. Thus in H.Pylori associated ITP if the treatment is initiated at the time of production of primary autoantibodies, the response to H.Pylori associated ITP treatment by triple therapy is good. At later stages of H.Pylori associated IT, when secondary and tertiary autoantibodies are formed, the disease then do not respond to triple therapy of H.Pylori eradication. H.Pylori associated ITP with "secondary" or "tertiary "autoantibody production probably should be treated with immunosuppressive agents like steroids, cyclophosphamide etc. along with triple therapy to eliminate the primary autoantibodies too if they remain still in advanced ITP associated with H.Pylori. Thus polyclonal, oligoclonal or monoclonal autoantibody production will command treatment of ITP. Early immunosuppression from drugs like Rituximab, steroids etc. or their combination can prevent clonal evolution and epitome spread in ITP patients.

$80 \%$ of ITP is "primary" and the rest $20 \%$ is "secondary". Causes of secondary ITP are varied, to mention a few -SLE(5\%),APS(2\%),CVID (1\%),EVAN syndrome(2\%), ALPS(1\%),Hepatitis C(2\%), H.Pylori(1\%), ITP post vaccination especially MMR(1\%) and various systemic infections(2\%). Since in primary ITP the cause is unknown, thus we lack specific treatment and in many patients we see the development of a "refractory adult ITP". In secondary ITP excellent responses are shown if the antigen triggering autoantibody production is withdrawn. Such good response to treatments with complete remission/ durable responses have been in secondary ITP like MMR, H. Pylori, CMV, VZV, Hepatitis C virus related ITP if the underlying disease has been treated well. Response is worse in ITP related to autoimmune diseases like EVANS syndrome, SLE, APS, ALPS. An intermediate response to treatment is observed among immunosupressed, post transplantation patients and in patients with CLL and CVID. ITP patients have antinuclear antibodies(incidence 15-25\%), antithyroid antibodies (incidence 25-50\%), antiphospholipid antibodies (incidence upto 30-40\%)suggesting further immune relation to the disease.

Certain secondary immune thrombocytopenias are drug induced. In drug induced thrombocytopenia, the platelet count decreases after ingestion of the drug and generally starts to reverse shortly after cessation of the drug. Quinidine, quinine, gold, heparin, sulphonamides can cause drug induced immune related thrombocytopenia. Sulphonamides cause early thrombocytopenia, whereas a long induction period is typical of gold salts. The mechanism proposed here is that the drug may conjugate with a platelet membrane and trigger production of hapten-dependent antibodies, or induction of a neoantigen which would later be recognized by circulating antibodies only in the presence of a drug.

\section{Conclusion}

Understanding of immunopathology of ITP is important to further manage these patients. Many patients with secondary ITP or even primary ITP can have long lasting remission from the disease if treated adequately. Elimination of antigen (if known for example Helicobacter Pylori) can cause complete remission of the disease / durable remissions of the disease. And in primary ITP, long complete durations can be achieved by administering steroids, intravenous immunoglobulins etc.. The meat of the matter here is to stop the production of autoantibodies / to eliminate the antigen triggering the autoimmune process. List of "secondary immune thrombocytopenias" will increase due to our better understanding of the disease, and less frequently will be these immune thrombocytopenias be categorized as "primary immune thrombocytopenias" in future. Disease specific therapies for secondary immune thrombocytopenias, and for primary immune thrombocytopenias - steroids and/or intravenous immunoglobulins are first line options, many a times resulting in complete remissions. Second and further line management of primary immune thrombocytopenias is done by TPO mimetics like eltrombopag or romiplostim, androgens - danazol, or by other immunosuppressive drugs like cyclophosphamide, vincristine, rituximab etc.

\section{References}

1. Rodeghiero F, Stasi R, Gernsheimer T, Michel M, Provan D, Arnold DM, etal.Standardization of terminology, definitions and outcome criteria in immune thrombocytopenic purpura of adults and children: report from an international working group. Blood 2009;113(11): 23862393. doi:10.1182/blood-2008-07-162503

2. Cines DB, Bussel JB, Liebman HA, Luning Prak ET. The ITP syndrome: pathogenic and clinical diversity. Blood 2009;113(26):6511- 6521. doi: 10.1182/blood-2009-01-129155

3. William J. Harrington, Virginia Minnich, James W. Hollingsworth, Carl V. Moore. Demonstration of a thrombocytopenic factor in the blood if patients with thrombocytopenic purpura. J lab.Clin.Med. 1951;38(1):1-10

4. Immune thrombocytopenia post infection: Molecular mimicry GP IIIa Nardis Met.al.PNAS(1997);94:7589 Zhang et. Al .Blood (2009);113:4086

5. Antonio Gasbarrini, Francesco Franceschi, Raffaele Tartaglione, Raffaele Landolfi, Paolo Pola, Giovanni Gasbarrini. Regression of autoimmune thrombocytopenia after eradication of Helicobactor Pylori. The Lancet. 1998;35:878. doi: 10.1016/S01406736(05)60004-9

6. Heterogeneity in ITP response to eradication of H.Pylori.Stasi et.al. Blood(2009)113:1231-1240 
7. Vyas Piyush, et al. Chemotherapy in a patient with metastatic duodenal cancer and idiopathic thrombocytopenic purpura: A case report and review of literature. J Cancer Prev Curr Res;4(6):00140. doi:10.15406/jcpcr.2016.04.00140

8. Czy maloplytkowosc moze byc przyczyna dyskwalifikacji od leczenia guzow litych.(Can thrombocytopenia be a reason to disqualify patients with solid tumours from treatments").e-poster presented at Polish Society of Haematologists and Transfusion medicine specialists 27TH Annual Conference. Vyas Piyush et al. .Acta Hematologica Polonica Elservier ;Suppl.1;Volume 48.2017

9. Piyush Vyas, Krzysztof Wózniak, Marta Dudek, Ewa Zurawiska Grzelka, Mateusz Cieslak, Anna Dryja. et al. Primary immune thrombocytopenia (ITP) and breast cancer. Case report and review of literature. 2017) Int J Hematol Blo Dis. 2017;2(2):1-5.

10. Semple JW. Immune pathophysiology of autoimmune thrombocytopenic purpura. Blood Rev.2002;16(1):9-12. doi 10.1054 /blre.2001.0172

11. Sakakura M, Wada H, Tawara I, Nobori T, Sugiyama T, Sagawa N. Reduced CD4+CD25+ $\mathrm{T}$ cells in patients with idiopathic thrombocytopenic purpura .Thromb. Res. 2007;120(2):187-193. doi: 10.1016/j.thromres.2006.09.008

12. Circulating dendritic cells subsets and CD4+FoxP3+ regulatory $\mathrm{T}$ cells in adult patients with chronic ITP before and after treatment with high dose dexamethasone .Yun Ling et.al.Eur J Haematol.2007:79(4):310-316

13. John W Semple. et al. ITP three 'R's: regulation, routing, rituximab. Blood .2008;112:927-928

14. Piyush Vyas, Krzysztof Wózniak, Marta Dudek, Ewa Zurawińska Grzelak, Leszek Kraj, Mateusz Cieślak. Chemotherapy in a patient with metastatic duodenal cancer and idiopathic Thrombocytopenic purpura: A case report and review of literature. J Cancer Prev Curr Res. 2016; 4(6):00140. doi: 10.15406/jcpcr.2016.04.00140
15. Piyush Vyas, Krzysztof Wózniak, Marta Dudek, Ewa Zurawiska Grzelka, Mateusz Cieslak, Anna Dryja. et al. Primary immune thrombocytopenia (ITP) and breast cancer. Case report and review of literature. 2017) Int J Hematol Blo Dis. 2017;2(2):1-5.

16. Czy maloplytkowosc moze byc przyczyna dyskwalifikacji od leczenia guzow litych?Eng.transplation: Can thrombocytopenia be a reason to disqualify patients with solid tumours from treating?(ePoster Annual conference of Polish society of haematologists and transfusion medicine specialists-2017.).Piyush Vyas et.al. Acta Hematologica Polonica Suppl1/Volume48-2017:35-36

17. Krauth MT, Puthenparambil J, Lechner K . Paraneoplastic autoimmune thrombocytopenia in solid tumors. Crit Rev Oncol Hematol. 2012;81(1):75-81. doi:10.1016/j.critrevonc.2011.02.004

18. Rossignol J, Michallet AS, Oberic L, Picard M, Garon A, Willekens C. et al. Rituximab-cyclophosphamide -dexamethasone(RCD) combination in the management of autoimmune cytopenias associated with chronic lymphocytic leukemia. Leukemia. 2011;16:2451. doi: 10.1038/leu.2010.278

19. Ekström Smedby K, Vajdic CM, Falster M, Engels EA, Martínez-Maza 0, Turner J. et al. Autoimmune disorders and risk of non Hodgkin lymphoma subtypes :a pooled analysis within the InterLymph Consortium. Karin Ekstrom Smedby et.al .Blood. 2008;111(8):40294038. doi: 10.1182/blood-2007-10-119974

20. Jeffrey J.Kirshner, Kenneth W.Zamkoff, Arlan J.Gottlieb. Case report Idiopathic thrombocytopenic purpura and Hodgkin's disease:report of two cases and a review of the literature.Jeffrey J .Kirshner et.al.The American Journal of the Medical Sciences. 1980;280(1):21-28. doi: 10.1097/00000441-198007000-00004

21. Michael Figueroa, Jane Gehlsen, Denis Hammond, Sharon Ondreyco, Lawrence Piro, Talisman Pomeroy. Combination chemotherapy in Refractory Immune Thrombocytopenic Purpura. New England Journal of Medicine. 1993;328:1226-1229. doi: 10.1056/ NEJM199304293281703 